\title{
¿CÓMO DARLE VISIBILIDAD A LA VIOLENCIA CONTRA LAS MUJERES EN CHILE? CONTRIBUCIÓN DE LAS AGENCIAS DE LAS NACIONES UNIDAS
}

\author{
MARÍA LUISA JÁUREGUI MEJÍA
}

(UNESCO, Chile)

\section{INTRODUCCIÓN}

Chile es un país atípico: por un lado se vive en la modernidad y prosperidad económica, pero por el otro, todavía existe una gran deuda pendiente, una deuda social. Esto se debe a que seguimos bajo la tutela de una Constitución que fue aprobada en 1980, durante la dictadura militar y que los cambios legales son lentos. Además existe una fuerte oposición de la Iglesia Católica a ciertos cambios legislativos, tales como la aprobación de una ley del divorcio, que se dilató hasta el año 2004, cuando se aprobó una Ley de Matrimonio Civil que considera el divorcio con muchos condicionantes.

Durante el Gobierno del Presidente Frei, se aprobó la Ley de Filiación, por la cual todos los hijos que nacen ahora son considerados legítimos y no «ilegítimos» o «naturales», como era el caso en años anteriores. También se ha aprobado una Ley Contra la Violencia Intrafamiliar, pero es necesario volverla a retomar para actualizarla y hacerla más eficaz, como veremos más adelante.

En Chile, el Congreso todavía no ha aprobado el Protocolo Facultativo de la CEDAW, de las Naciones Unidas, que obliga a respetar, proteger y garantizar los derechos de la mujer. La Convención de Belem do Pará de la Organización de los Estados Americanos aprobada en 1994, fue ratificada por el Estado Chileno en 1996 y oficializada en 1998, pero esta aprobación ha tenido un impacto bastante reducido en la sociedad. Se han hecho grandes esfuerzos para capacitar a la policía y crear comisarías especiales para que traten la violencia intrafamiliar, pero se admite que estos esfuerzos son aún limitados.

Los últimos estudios conocidos sobre la violencia contra las mujeres datan del año 2001. Según un estudio realizado ese año por el Servicio Nacional de la Mujer (SERNAM) y del Centro de Análisis de Políticas Públicas de la Universidad de Chile, en la región metropolitana (Santiago de Chile), entre las mujeres que tienen entre 15 y 49 años de edad, un 50,3 \% de las mujeres ha vivido 
alguna vez violencia en la relación de pareja. De ellas, un 34,1\% ha sufrido violencia física y/o sexual y un 16,3 \% ha sufrido violencia psicológica. ${ }^{1}$

En ese mismo año (2001), de los 7.129 procesos que se examinaron en esa época, por violencia intrafamiliar, sólo el 9,7 \% tuvo sentencia condenatoria. ${ }^{2}$

Por lo que se refiere al trabajo en Chile, sólo declara trabajar un $43 \%$ de las mujeres y ganan alrededor del $63 \%$ de la remuneración de los hombres con iguales calificaciones. El 27,7 \% de las trabajadoras dependientes no tiene contrato.

El acoso sexual afecta a un tercio de las empleadas de oficina; al $25 \%$ de las mujeres que trabajan en la industria y a un $15 \%$ de las trabajadoras no calificadas. El Proyecto de Ley sobre el Acoso Sexual sigue pendiente de aprobación en el Congreso. ${ }^{3}$

Frente a esta situación, el SERNAM, con estatuto ministerial, solicitó a las Agencias de las Naciones Unidas a que le apoyaran para hacer visibles los temas de la violencia y abuso sexual en Chile, en el marco de unas «Mesas de Convivencia Democrática» creadas en el año 2001, con la participación de instituciones estatales y de la sociedad civil.

En este artículo se presentarán las intervenciones realizadas por cinco Agencias de las Naciones Unidas en el marco de la Mesa Temática de Género, que agrupó a los Puntos Focales de Género o Especialistas en Género de esas Agencias $^{4}$ incluyendo a la UNESCO. El trabajo se realizó en los años 2002 a 2004, con el principal objetivo de dar a conocer la violencia contra las niñas y mujeres y proponer recomendaciones para la elaboración de políticas públicas.

La UNESCO trabaja desde hace años para promover una convivencia pacífica en el marco de sus actividades que responden al Decenio de las Naciones Unidas por una Cultura de Paz y No Violencia contra los Niños del Mundo. Esto lo realiza en el campo educativo, fomentando la convivencia, los valores democráticos, la importancia de reconocer la diversidad de manera positiva, la innovación y la lucha contra la exclusión y cualquier otra forma de discriminación.

Entre sus programas cabe citar el trabajo con las 1.200 escuelas en la región que pertenecen a una Red de Escuelas Asociadas a la UNESCO, el Programa

1. CEPAL: Violencia contra la mujer en relación de pareja: América Latina y el Caribe. Una propuesta para medir su magnitud y evolución por Diane Alméras, Rosa Bravo, Vivian Milosavljevic, Sonia Montaño y María Nieves Rico, de la Unidad Mujer y Desarrollo de CEPAL, Documento n 40 , Serie Mujer y Desarrollo. Proyecto Interagencial «Uso de Indicadores de Género para la Formulación de Políticas Públicas». Vid. Cuadro 2. «Encuestas para medir la violencia contra la mujer llevadas a cabo en América Latina y el Caribe», Santiago de Chile, junio de 2002.

2. OXFAM, PNUD, ACTIVA: Equidad de Género: Condición de Democracia en el Manual para el Control Ciudadano de la Declaración del Milenio. Pobreza y Equidad de Género, Módulo 10, Santiago de Chile, 2003.

3. OXFAM, PNUD, ACTIVA: Equidad de Género: El Camino Recorrido en el Manual para el Control Ciudadano de la Declaración del Milenio. Pobreza y Equidad de Género, Módulo 7, Santiago de Chile, 2003.

4. Fondo de Naciones Unidas para la Población (FNUAP), Organización Mundial para la Salud (OPS), Programa de las Naciones Unidas para el Desarrollo (PNUD), Agencia de las Naciones Unidas para la Educación, la Ciencia y la Cultura (UNESCO), Fondo de las Naciones Unidas para los Niños (UNICEF) y Agencia de las Naciones Unidas para la Mujer (UNIFEM). 
¿Cómo darle visibilidad a la violencia contra las mujeres en Chile? Contribución de...

INNOVEMOS de innovaciones educativas o en la formación de directivos y docentes a través de sus redes (Ver el Portal Institucional: www.unesco.cl).

\section{DEFINICIONES DE VIOLENCIA, ALGUNOS ANTECEDENTES}

Un hito importante dentro del marco jurídico internacional lo constituye la creación de una "Convención sobre la eliminación de todas las formas de discriminación contra la mujer», conocida como CEDAW, por sus siglas en inglés, aprobada el 18 de diciembre de 1979.

En nuestra región, la "Convención Interamericana para Prevenir, Sancionar y Erradicar la Violencia contra la Mujer» (mejor conocida como Convención de Belem do Pará) fue adoptada por la Asamblea General de la Organización de los Estados Americanos el 9 de junio de 1994.

Estas Convenciones, más las Conferencias Mundiales organizadas por las Naciones Unidas en Viena (1993), Cairo (1994) y Beijing (1995), ayudaron a que muchos países de nuestra región promulgaran leyes contra la violencia doméstica y/o la violencia familiar durante la década de los noventa.

El nombramiento de la Relatora Especial sobre Violencia contra la mujer, en 1994, es también un hecho importante que ha permitido la visibilidad de distintas formas de violencia contra las mujeres.

Según la Convención de Belem do Pará, se entiende por violencia contra la mujer "cualquier acción o conducta basada en su género, que cause muerte, daño o sufrimiento físico, sexual o psicológico a la mujer, tanto en el ámbito público como en el privado». (art. 1)

También

«que la violencia contra la mujer incluye la violencia física, sexual o psicológica: que tenga lugar dentro de la familia o unidad doméstica o en cualquier otra relación interpersonal, ya sea que el agresor comparta o haya compartido el mismo domicilio que la mujer, y que comprende, entre otros, violación, maltrato y abuso sexual; que tenga lugar en la comunidad y sea perpetrada por cualquier persona y que comprende, entre otros, violación, abuso sexual, tortura, trata de personas, prostitución forzada, secuestro y acoso sexual en el lugar de trabajo, así como en instituciones educativas, establecimientos de salud o cualquier otro lugar, y que sea perpetrada o tolerada por el Estado o sus agentes, dondequiera que ocurra». (art.2)

Sin embargo, sabemos que no basta con contar con declaraciones y convenciones, pues como lo indicaba Mary Robinson, la Alta Comisionada de los Derechos Humanos:

«Las mujeres alrededor del mundo han encontrado que solamente declaraciones y convenciones no son garantía suficiente de sus derechos humanos. Ya es tiempo de traducir finas palabras en firmes acciones por parte de las organizaciones internacionales, nacionales y gobiernos locales, junto con la sociedad civil y que ello asegure que los derechos de las mujeres se honren plenamente en todas partes». ${ }^{5}$

5. Extractos de un Mensaje de Mary Robinson en el Día de la Mujer. 
Con el paso del tiempo se ha acuñado definiciones más extremas cuando se quiere referir a las mujeres que mueren por causas de violencia, se trata del término de "femicidio", según definiciones conceptuales de las autoras Diana Russell y Hill Radford ${ }^{6}$. En este caso «femicidio» califica la muerte de las mujeres dentro o fuera del hogar, ejercida por los hombres en su deseo de ejercer sobre ellas poder, dominación o control.

El término «femicidio» busca hacer visible la violencia contra las mujeres más que la violencia intrafamiliar, o violencia doméstica, ejercida contra niños, niñas y mujeres, que es la que se contempla en la mayoría de las leyes aprobadas en los países de la región, lo cual, según muchas activistas feministas ${ }^{7}$, está diluyendo la mirada de la violencia ejercida contra las mujeres.

\section{EL TRABAJO DE LAS AGENCIAS EN CHILE}

\subsection{Trabajo de Prevención de la Violencia Intrafamiliar}

La Mesa Temática Interagencial de Género y el SERNAM decidieron comenzar en el año 2002, con el tema de la prevención de la violencia intrafamiliar. En el año 2003, se tomó el tema del tratamiento de la violencia contra las mujeres y en el año 2004 se publicó un estudio sobre «el femicidio en Chile», es decir que se pasó de la prevención de la violencia, al tratamiento de la violencia y finalmente a la denuncia de la violencia.

El primer gran tema que se abordó en el año 2002 fue el de hacer visible experiencias validadas de convivencia democrática en función de la prevención de la violencia contra niños, niñas, adolescentes y jóvenes, hombres y mujeres, y recoger de ellas los factores que tienen una incidencia favorable en el desarrollo psicosocial de sus destinatarios y de allí hacer recomendaciones para la elaboración de políticas y programas públicos.

Se decidió realizar tres jornadas de trabajo en las cuales se analizó y sistematizó experiencias de distintas instituciones según las etapas del ciclo vital: infancia, adolescencia y juventud. Al término del trabajo se publicó la sistematización realizada con el fin de lograr una estrategia de prevención de la violencia intrafamiliar a partir de las experiencias presentadas ${ }^{8}$.

6. Russell, Diana y RADFord, Hill: Femicide: the Politics of Woman Killing, New York, Twayne Publishers, 1992.

7. En los ámbitos académicos y movimientos feministas, fundamentalmente de América del Sur, se defiende la importancia de diferenciar el término «Femicidio» del de "Feminicidio». El concepto de feminicidio (genocidio contra las mujeres) define el acto de asesinar a una mujer por el mero hecho de pertenecer al sexo femenino. Feministas como Ana $\mathrm{M}^{a}$ Portugal o Marcela Lagarde definen el término como más político, ya que en él se incluye no sólo al agresor individual sino que se apela a la existencia de una estructura estatal y judicial que avala estos crímenes. Uno de los ejemplos más emblemáticos del Feminicidio son las llamadas «muertas de Juárez». Se trata de más de 400 mujeres asesinadas desde 1993 después de haber sido secuestradas, violadas y torturadas.

8. Gobierno de Chile, OPS/OMS, UNFPA: Sistematización de Experiencias de Prevención de Violencia Intrafamiliar y Abuso Sexual, Proyecto «Mesas de Convivencia democrática», Documento de trabajo elaborado por Flor Draguicevic P. Santiago de Chile, Noviembre-Diciembre 2002. 
¿Cómo darle visibilidad a la violencia contra las mujeres en Chile? Contribución de...

Cada una de las Agencias de las Naciones Unidas participó en la selección de los programas que debían presentarse y se encargó de moderar una de las tres jornadas. Participaron todas las Agencias en la sistematización que se realizó al término del trabajo para su publicación.

La UNESCO lideró las jornadas sobre adolescencia e invitó a participar a tres experiencias: Los Comités de Convivencia Democrática del Ministerio de Educación; la Escuela Agroecológica de Pirque y la Escuela Valle de Lluta.

Los Comités de Convivencia Democrática del Ministerio de Educación de Chile buscan cambiar la cultura escolar promoviendo la apertura a través de una red de profesores abiertos al cambio. Se revisan las normas de los establecimientos y se buscan procedimientos para la resolución no violenta de conflictos a partir de la visión y la misión de los proyectos educativos. Se rompe con el autoritarismo mediante prácticas horizontales y se buscan espacios de diálogo y de construcción.

La Escuela Agroecológica es una escuela que promueve el diálogo horizontal a todo nivel basándose en un acercamiento ecológico y en una ecología interior que fomenta la meditación, el respeto a la espiritualidad y el conocimiento de sí mismo. Esta Escuela técnica y agrícola da mucha importancia a la negociación y favorece una visión holística, integrada e integral.

La Escuela de Valle de Lluta pertenece a la Red de Escuelas Asociadas a la UNESCO en Chile, es una escuela que promueve la mediación entre pares. El trabajo es voluntario y participan en el programa los niños y niñas que así lo desean. El trabajo ha dado muy buenos resultados bajando el nivel de violencia en el aula y en las horas de recreación. Además la escuela trabaja con los padres y madres de los alumnos, de escaso nivel educativo, ofreciendo programas de alfabetización.

Los programas presentados en las jornadas del año 2002 mostraron líneas de acción desarrolladas en el ámbito pre-escolar y escolar. De los nueve programas presentados sólo tres incluían la dimensión de sexualidad y cinco la dimensión de género en las estrategias que implementaban con niños, niñas y jóvenes de ambos sexos.

Las experiencias analizadas demostraron que existe una desarticulación en el sistema escolar. Mientras en los niveles pre-escolar y escolar se implementan líneas de trabajo que promueven cambios de conductas, de comportamientos no violentos, y se logra trabajar con la comunidad educativa y local, en los grados de enseñanza secundaria y superior no ocurre lo mismo.

En los niveles de educación secundaria y superior existen mallas curriculares más rígidas y se da poca o nula importancia a la socialización o a la resolución no violenta de conflictos. En estos niveles se entregan modelos de aprendizaje con estereotipos culturales que lamentablemente perpetúan las mismas condiciones de una sociedad rígida y autoritaria. Esta situación dificulta la introducción de iniciativas innovadoras que fomenten una convivencia pacífica lo cual no permite producir cambios significativos en la conducta de los docentes y educandos. 
Entre las recomendaciones que se hicieron al terminar estas jornadas se trató el tema de un esfuerzo sostenido para introducir la educación sexual en la escuela; la educación para la resolución no violenta de conflictos; el aprendizaje de los Derechos Humanos a través de una nueva pedagogía que cuestione las prácticas autoritarias que aún persisten en la cultura escolar chilena.

En el año 2004 se notó un incremento de la violencia en las escuelas, lo que ha obligado al Ministerio de Educación de tratar la introducción de la resolución de conflictos en la escuela de manera más sistemática.

\subsection{El tratamiento de las mujeres que han sufrido violencia}

En el año 2003, se siguió el modelo anterior de seleccionar programas de interés y presentarlos en jornadas, al mismo tiempo que se sistematizaban los programas presentados y los resultados de las discusiones.

A diferencia de las jornadas anteriores, en el año 2003 se hizo un esfuerzo para realizar las tres jornadas de trabajo en distintas zonas geográficas del país, norte (Arica), sur (Puerto Montt) y centro (Santiago), con el fin de permitir a un mayor participación y representatividad en el número de instituciones estatales y de la sociedad civil. La UNESCO se encargó de la jornada realizada en Santiago.

Además, en la organización de estas jornadas no sólo participó el SERNAM, si no también el Ministerio de Salud y la Red Chilena contra la Violencia Doméstica y Sexual.

Participaron las mismas Agencias de Naciones Unidas que habían trabajado la prevención en el año 2002 .

En las tres jornadas se abordó el tema de la violencia contra la mujer en la región y los acuerdos establecidos en las convenciones de las Naciones Unidas (CEDAW) y de la OEA (Convención de Belem do Pará). La Red Social presentó los problemas desde el punto de vista de sus actividades en la temática y el SERNAM y el Ministerio de Salud presentaron los programas y líneas de acción a nivel nacional tanto en lo conceptual, como en lo legislativo y en los modelos de intervención, como el Proyecto Piloto de Intervención en Violencia Intrafamiliar en la Atención Primaria.

Este proyecto se desarrolla en dos consultorios de la región Metropolitana mediante un convenio entre los dos ministerios. El programa piloto se ha definido con un enfoque integral y familiar, orientado a mejorar la calidad de vida de las personas, las familias y las comunidades y se considera como uno de los planes fundamentales para el logro del objetivo de la reforma del sector salud.

En estas jornadas se presentaron nueve experiencias y se contó con la participación de representantes de diversas instituciones gubernamentales y de la sociedad civil, con quiénes se pudo discutir sobre las intervenciones que se hacían, sus logros y limitaciones, que luego fueron sistematizados.

Entre las experiencias que se presentaron había campañas radiales contra la violencia hacia las mujeres (en el norte) que tienen por objeto prevenir la violencia en Calama y Chuquicamata, ciudades reconocidas por la magnitud del problema de violencia intrafamiliar (VIF), producto de una cultura machista 
¿Cómo darle visibilidad a la violencia contra las mujeres en Chile? Contribución de...

existente en estas ciudades mineras. Otra iniciativa similar es la realizada en Coronel, donde una ONG, el Grupo Proceso, se encargó de la sensibilización y prevención de la VIF mediante campañas radiales y otras actividades.

También se refirió a líneas de asistencia psicosocial y legal en la prevención de la violencia doméstica y sexual como la realizada por la ONG Casa de Encuentro de la Mujer en Arica. Su principal acción es el acompañamiento de las víctimas de la VIF.

Otras iniciativas que se presentaron en las jornadas fueron las actividades realizadas por los Centros Comunitarios en donde agrupaciones de mujeres, administradas por ONGs o apoyadas por el Ministerio de Salud, organizan la acogida transitoria o se constituyen en Casas de Protección para las mujeres y sus hijos que han sido víctimas de violencia, realizando así mismo apoyo psicológico, social y legal.

Estas Casas están ubicadas en distintas zonas del país. Entre ellas se encuentran el Centro Mirabal (ONG Pachamama) en la Comuna de Coronel, en el norte del país; los Centros Comunitarios de Salud Mental y Familiar La Rueda en Temuco, en el sur del país y en Pudahuel en Santiago, ambos apoyados por el Ministerio de Salud. La Casa Andrea Araneda, de primera acogida en violencia, que es un proyecto municipal en La Bandera, San Ramón en Santiago. Nueva Mujer, un programa de la Unidad de Salud Mental del Hospital Las Higueras de Talcahuano en el sur del país.

Entre las principales lecciones aprendidas ${ }^{9}$ que aparecen en la sistematización, está la falta de conocimiento, por parte de la comunidad, sobre sus derechos en materia de violencia intrafamiliar. A pesar de que, como se ha visto, ya existe una Ley Contra la Violencia Intrafamiliar, todavía no se conoce su alcance. Las mujeres desconocen las leyes y derechos que las protegen y desconocen los mecanismos de denuncia y protección o el ejercicio y la exigibilidad de los mismos. Esto da como resultado una inexistente o débil participación social en la evaluación y control de las políticas públicas en la materia.

Entre las recomendaciones que hicieron los agentes comunitarios en estas jornadas de discusión fue la de buscar estrategias de capacitación de agentes comunitarios, líderes y promotores en leyes y derechos de las personas víctimas de violencia. La capacitación de estos agentes debía poner especial atención en el conocimiento de los procedimientos de la normativa jurídica más actualizada.

Si bien es cierto que muchas de las iniciativas presentadas mencionaban la capacitación de sus agentes, los procesos de capacitación no han sido evaluados o sistematizados por lo que es muy difícil juzgar el impacto de los mismos. Además está el problema del financiamiento que no siempre se puede sostener en el tiempo, dejando muchas veces a las víctimas de violencia en desamparo, por necesidad de cerrar los centros de acogida. Para ello se sugirió la creación

9. DragiCEVIC, Flor: Resumen Ejecutivo Jornadas de «Prevención en Violencia Intrafamiliar»: Ámbito Salud, Santiago de Chile, 2004. Documento aún no publicado. 
de redes comunales y el establecimiento de conexiones con distintas instancias gubernamentales, privadas y no gubernamentales para garantizar el éxito de las intervenciones.

Los Centros de Salud que pertenecen al Proyecto Piloto de Intervención en Violencia Intrafamiliar en la Atención Primaria de los Ministerios de Salud y SERNAM han logrado diseñar y validar los instrumentos siguientes: Detección de la Violencia; Diagnóstico de Gravedad; Protocolo de Fase Diagnóstica y de Intervención de Primer Orden Individual; Intervención en Violencia Leve y Moderada y un Protocolo de Atención en Violencia a la Pareja de las Mujeres.

El problema con este proyecto es que hasta ahora ha dependido del Programa de Depresión del Ministerio de Salud y debe esperar que se instale un Programa de VIF autónomo para poder extenderse al resto del país.

\subsection{La denuncia de la violencia contra las mujeres}

El año 2004 la Mesa Temática Interagencial de Género encomendó un estudio sobre el femicidio en Chile ${ }^{10}$ a la Corporación La Morada. Los resultados más impactantes del estudio, son aquellos que evidenciaron la imposibilidad de identificar en los sistemas de registro existentes si una mujer asesinada había denunciado en alguna oportunidad la violencia que la afectaba. En efecto, los registros de denuncias por VIF no permiten identificar a las mujeres denunciantes, ni saber si han sido asesinadas. Asimismo, los juicios por homicidio de mujeres no incorporan información sobre denuncias anteriores, por lo que existen vacíos legales que se deben corregir.

Los sistemas de registro, tanto en las unidades policiales, juzgados del crimen o en los tribunales civiles, que son los que aplican la Ley para la Violencia Intrafamiliar, son diferentes y obedecen a principios distintos. El estudio realizado por la Mesa temática Integral de Género permite constatar la imposibilidad de construir una información que dé cuenta de la magnitud real del femicidio en Chile. En efecto, la ausencia de información estadística respecto del sexo de las víctimas y del vínculo con el agresor, impide identificar cuándo se trata de mujeres asesinadas por razones de género.

La revisión de expedientes judiciales de homicidios de mujeres y la prensa escrita, en particular el diario sensacionalista La Cuarta, posibilitó construir una cuantificación de los hechos que, aun siendo parcial, da cuenta de la existencia del fenómeno y permite reconocer sus principales características, pero no atiende la situación con la urgencia y gravedad que se requiere. Además, la manera de presentar los hechos de sangre deja mucho que desear, son sumamente sesgados, y constatan que no existe una sensibilidad sobre el tema instalada en la prensa chilena. A menudo se justifican los malos tratos y los delitos de sangre invocando los celos u otros comportamientos típicamente machistas.

10. NACIONES UNIDAS: Femicidio en Chile, Estudio Realizado por el Área de Ciudadanía y Derechos Humanos de la Corporación de La Morada, Santiago de Chile, Octubre de 2004. 
¿Cómo darle visibilidad a la violencia contra las mujeres en Chile? Contribución de...

Las fugas de información y los subregistros detectados en las distintas instancias institucionales responsables de los procedimientos policiales, judiciales y médicos, no permiten reconocer las características de estos asesinatos. Datos clave para identificar el femicidio tales como el vínculo entre las mujeres asesinadas y sus victimarios, las circunstancias y los móviles de estos crímenes, así como los antecedentes de violencia, no son considerados relevantes por las instituciones y funcionarios que intervienen a lo largo del proceso, lo que da como resultado su invisibilidad y por tanto la ausencia de prevención y de atención al fenómeno en el país.

\section{CONCLUSIÓN}

A finales del año 2004, la Mesa Temática Interagencial de Género realizó un balance de las actividades realizadas desde el año 2002 en el marco de las Mesas por un Convivencia Democrática creadas por el SERNAM. Allí se constató que se había trabajado con varias instancias gubernamentales y de la sociedad civil, para examinar las actividades que estaban siendo promovidas en los ámbitos de la prevención, tratamiento y denuncia de la violencia contra las mujeres, y que era necesario seguir en el empeño de incidir en la elaboración de las políticas públicas del país para remediar esta situación.

Como hemos visto, en el primer año se trabajó la prevención y la vinculación de los programas del sistema formal escolar en donde se constató que sigue siendo necesario prever intervenciones más puntuales que tomen en cuenta el mejoramiento de la entrega de programas de educación sexual, resolución no violenta de conflictos, e introducción de la enseñanza de los Derechos Humanos, entre otros.

En el segundo año se examinaron las políticas en el tema de salud para el tratamiento de las víctimas de violencia, con ofertas desde las ONG, las organizaciones de auto-ayuda, las Casas de acogida de las víctimas de violencia, el programa piloto del SERNAM y del Ministerio de Salud, que entrega atención primaria. Se constató que existen vacíos en el tratamiento de la atención, que sólo se está considerando la violencia leve pero que no hay reales previsiones para la violencia grave y el abuso sexual.

El estudio sobre el femicidio en Chile, que fue publicado y dado a conocer en el año 2004, demostró los vacíos existentes en materia de recogida de datos fiables para documentar los casos graves de violencia contra las mujeres como es el femicidio y en donde todos tenemos la responsabilidad de colaborar, para mejorar la difícil coordinación entre las distintas instituciones que intervienen.

Con estos datos se concitó una cita con la Ministra del SERNAM que se llevó a cabo en febrero de 2005, en donde se revisaron los resultados del trabajo de la Mesa Temática Interagencial de Género y se decidió seguir trabajando para que se pueda apoyar a la nueva Comisión Intersectorial de Violencia, creada por el SERNAM para evaluar el rol, la influencia y el efecto de la legislación existente en Chile en materia de violencia contra la mujer, ya que es preciso sacar el tema del ámbito privado y colocarlo en el ámbito de preocupación del Estado y de la sociedad. 
Una de las prioridades actuales es actualizar el Programa Nacional de Prevención de la Violencia Intrafamiliar del SERNAM y la Ley de Violencia Intrafamiliar, actualmente en el Senado.

Éstas contemplan una reforma judicial familiar que ayude a resolver leyes de procedimiento, acceso más efectivo a la justicia, sanciones más fuertes a los victimarios. Se crea la figura de delito de maltrato habitual, se extiende la condena de los victimarios y se obliga a los jueces a considerar la aplicación de medidas cautelares o de protección a las víctimas hasta que se resuelva el caso; se crean vínculos más apropiados con la nueva legislación en el marco de la Reforma Procesal Penal, donde no se deja al criterio de los jueces cuestiones que ya han sido juzgadas. También se crean vínculos con la Ley de Tribunal de la Familia que ha sido aprobada recientemente.

Esta reforma busca la aplicación de justicia frente a situaciones de violencia, ya que la antigua ley no ha respondido a las nuevas expresiones de violencia contra las mujeres. A pesar de la capacitación que se ha realizado - en violencia intrafamiliar a la policía, por ejemplo, en la práctica todavía existen brechas culturales fuertes, rechazo social, político y legal. Lo que se desea es crear por primera vez una topología penal de maltrato familiar y hacer a las mujeres más conscientes de su derecho a denunciar el maltrato. Se busca un tratamiento más integral que contemple la prevención, la atención y que reconozca el rol de coordinación del SERNAM en esta materia.

La Ministra del SERNAM ha solicitado a las Agencias de las Naciones Unidas que participan en la Mesa Interagencial Temática de Género que le demos nuestro apoyo a su Agenda Legislativa para reforzar esta visión integral, para que la actual reforma de la Ley de Violencia Intrafamiliar responda a las inquietudes que han sido detectadas en nuestro trabajo de estos tres años.

\section{BIBLIOGRAFÍA}

CEPAL: Violencia contra la mujer en relación de pareja: América Latina y el Caribe. Una propuesta para medir su magnitud y evolución por Diane Alméras, Rosa Bravo, Vivian Milosavljevic, Sonia Montaño y María Nieves Rico de la Unidad Mujer y Desarrollo, Documento $n^{\circ} 30$, Serie Mujer y Desarrollo, Proyecto Interagencial «Uso de Indicadores de Género para la Formulación de Políticas Públicas», Santiago de Chile, junio de 2002.

DRAGICEVIC, Flor: Resumen Ejecutivo Jornadas de "Prevención en Violencia Intrafamiliar»: Ámbito Salud, Santiago de Chile, 2004. Documento aún no publicado.

GOBIERNO DE CHILE, OPS/OMS/UNFPA: Sistematización de experiencias de Prevención de Violencia Intrafamiliar y Abuso Sexual, Proyecto "Mesas de Convivencia Democrática», Documento de trabajo elaborado por Flor Draguicevic, Santiago de Chile, Noviembre-Diciembre 2002.

NACIONES UNIDAS: Femicidio en Chile, Estudio realizado pro el Área de Ciudadanía y Derechos Humanos de la Corporación de la Morada, Santiago de Chile. Octubre 2004.

OXFAM, PNUD, ACTIVA: Equidad de Género: El Camino Recorrido en el Manual para el Control Ciudadano de la Declaración del Milenio. Pobreza y Equidad de Género, Módulo 7, Santiago de Chile, 2003. 
¿Cómo darle visibilidad a la violencia contra las mujeres en Chile? Contribución de...

OXFAM, PNUD, ACTIVA. Equidad de Género: Condición de Democracia en el Manual para el Control Ciudadano de la Declaración del Milenio. Pobreza y Equidad de Género, Módulo 10, Santiago de Chile, 2003.

Russell, Diana y RADFORD, Hill: Femicide: the Politics of Woman Killing, New York, Twayne Publishers, 1992. 